\title{
Study on the giant magnetostrictive actuator control strategy based on genetic algorithm setting the PID parameter
}

\author{
Dengyuan Mo 1,a , Qidou Zhou ${ }^{2}$, Shaochun Ding ${ }^{3}$, Zhiyong Xie ${ }^{2}$ \\ ${ }^{1}$ Naval University of Engineering, College of Power Engineering, 430033, Wuhan, Province Hubei, China \\ ${ }^{2}$ Naval University of Engineering, Faculty of Naval Vessel Engineering, 430033, Wuhan, Province Hubei, China \\ ${ }^{3}$ Naval University of Engineering, Department of Science R\&D, 430033, Wuhan, Province Hubei, China
}

Keywords: Giant magnetostrictive actuator (GMA); Mathematical model; PID parameter setting; Genetic algorithm (GA); Simulation analysis

\begin{abstract}
In This paper, the relevant research is carried out about seabed sound reflection . It has influences on the test results of the real-boat test based on the acoustic holographic technology. The effects of seabed reflection on underwater acoustic test is analyzed, through simulating sound field of free field and semi-free field are seabed with different absorption capacity. By comparing the amplitude and phase relative errors under the free field for the acoustic test is determined, and the upper frequency limit is discussed. The basis for sitting sea trials used to do real-boat test based on the acoustic holographic technology and optimizing test program are proposed.
\end{abstract}

\section{Introduction}

Giant magnetostrictive actuator is very superior in all aspects of the performance, which makes it has good application prospect in various fields. In the process of application of actuators, the most important thing is to control them effectively, make it achieve its function according to the idea of the user. Giant magnetostrictive material is a kind of ferromagnetic materials, so it has certain hysteresis characteristics, and the permanent magnet, yoke material of actuator itself also exist hysteresis characteristics [1]. It makes the output of the actuator have a serious hysteresis and nonlinear, affect its wide application.

Since the 1940s, the PID controller was brought up, through continuous improvement, it has become the most widely industrial application of a kind of control method. And it is also the oldest and most mature control strategies. After investigation statistics, the PID control method and optimal PID control the proportion is as high as $90 \%$ in the field of industrial control method. And in the process of using PID control, the three parameters setting is the key step [2], traditional way of setting is often through the manual setting that is difficult to meet the needs of system. This paper proposes control strategy of genetic algorithm tuning PID parameters which improve the accuracy and efficiency of giant magnetostrictive actuator control. $\mathrm{Ri}$ is the distance between the real sound source $\mathrm{S}$ and the measuring point $\mathrm{P}$. $\mathrm{Rr}$ is the distance between the virtual sound source $\mathrm{S}$ ' and the measuring point $P$. 


\section{The basic principle of GMA and its control system mathematical model}

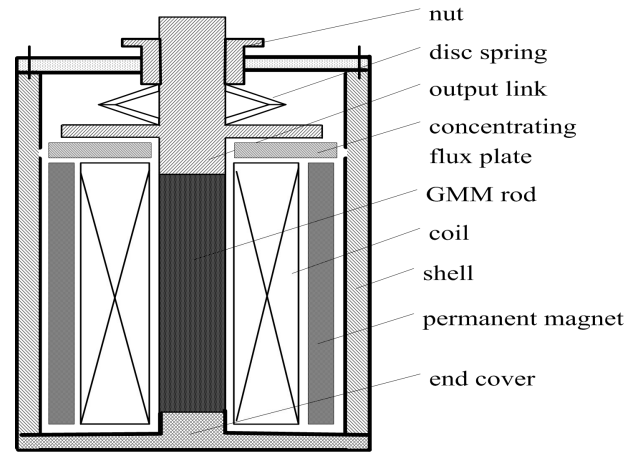

Figure 1. The simple structure diagram of GMA

Figure1 is the simple structure diagram of GMA. The work basic principle of GMA is: After the ventilation with alternating current $(\mathrm{ac})$, drive coil produces the changing magnetic field effect in magnetostrictive rod. Due to the magnetostriction effect, giant magnetostrictive rod is telescopic, thus it can export displacement and force. The mainly effect of bias magnetic field is that eliminating frequency doubling phenomena of the giant magnetostrictive rod to let the output with same frequency. Adjusting the size of the input alternating current can be achieved on the control of output displacement and output force of actuator [3].

Figure 2 is dynamic model of actuator.

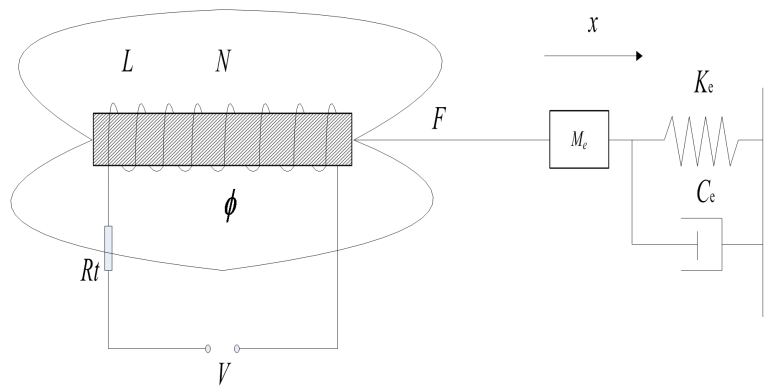

Figure 2. Dynamic model of actuator

To control the actuator, mathematical model must be established. As it has been in motion when the actuator works, it must write the equations of motion. This article will be established equations of motion by known physical formula, which is based on the physical phenomena.Through theoretical derivation, the transfer function block of actuator can be obtained, as shown in Figure 3. 


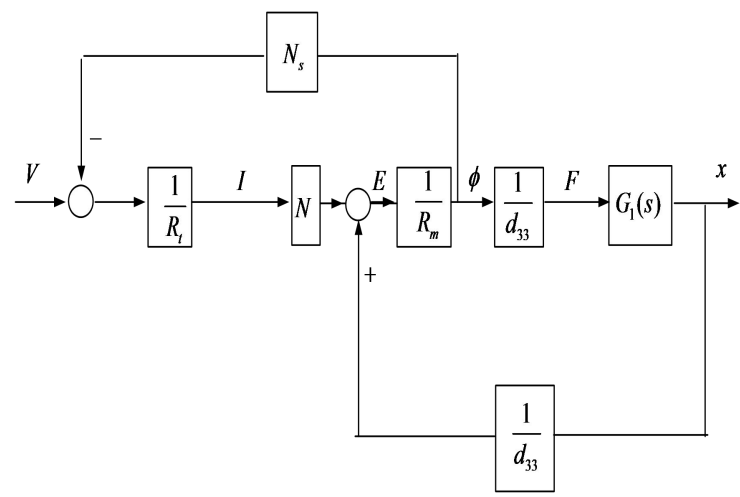

Figure 3. The transfer function block of actuator

Therefore, the actuator transfer function is:

$$
G(s)=\frac{x(s)}{V(s)}=\frac{N d_{33} \omega_{n}^{2}}{(L s+R)\left[k d_{33}^{2} R_{m}\left(s^{2}+2 \xi \omega_{n} s+\omega_{n}^{2}\right)-\omega_{n}^{2}\right]}
$$

\section{Genetic algorithm tuning PID parameters}

PID control algorithm adapts to a linear system [4], and it is a negative feedback control algorithm. Comparing the target and the output value, exert proportional control, integral control and differential control to the difference between the two comprises, in order to achieve control of the system. Figure 4 is the PID controller schematics.

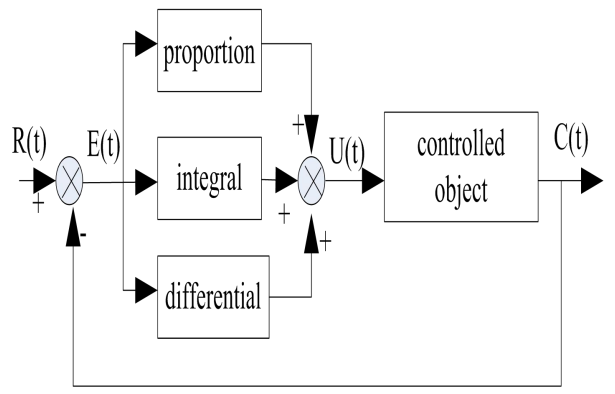

Figure 4. The simple structure diagram of GMA

The computing equation of PID control algorithm [5] for is:

$$
u(t)=K_{p}\left[e(t)+\frac{1}{T_{i}} \int_{0}^{t} e(t) d t+T_{D} \frac{d e(t)}{d t}\right]
$$

Wherein, $u(t)$ is the control signal, $K_{p}$ is the proportional coefficient, $T_{i} T_{i}$ is the integral constant, $T_{D}$ is the differential constant. 
In For PID controllers, the proportional coefficient, integral coefficient and differential coefficient tuning is critical, only to find the appropriate parameters in order to make the system stable, so as to achieve good control effect.

The method of genetic algorithm tuning PID parameters [6], namely genetic algorithms to generate an initial set of parameters given PID controller, then the system is running to get an output value. Comparing the output value and the expected value, through genetic algorithm automatically merit function continuously optimize the parameters entered into the PID controller, and finally get a set of parameters closest expectations, the specific process shown in Figure 5.

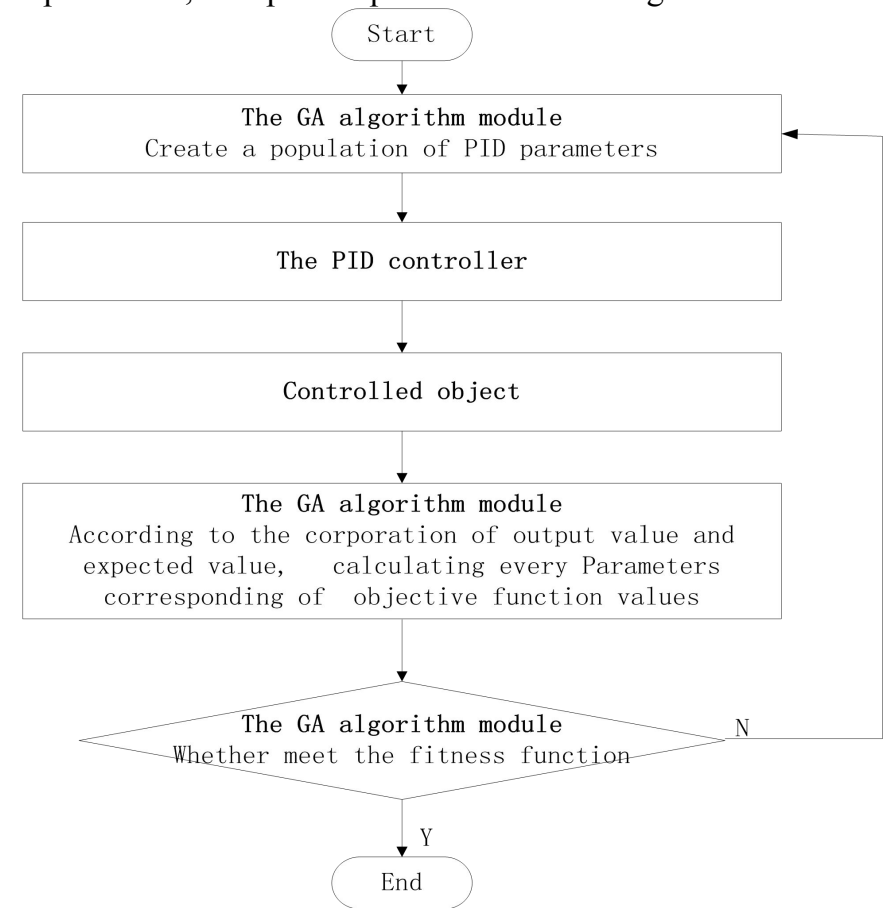

Figure 5. Genetic algorithm tuning PID parameters flowchart

For a real boat, is The method of genetic algorithm tuning PID parameters are mainly includes three parts: GA algorithm module, PID controller and controlled object. Which, PID controller parameters is given by GA algorithm [7], rather than manual tuning.

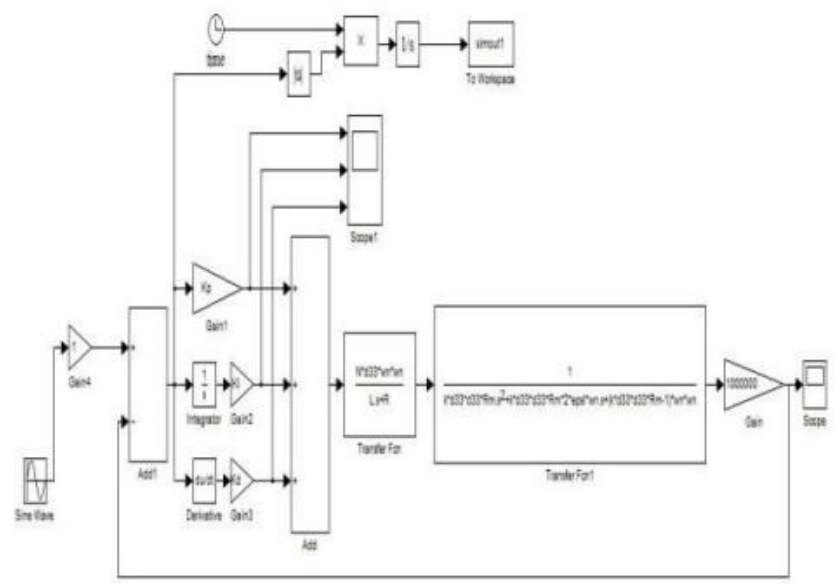

Figure 6. The genetic algorithm tuning PID parameters control chart 
Figure 6 is genetic algorithm tuning PID parameters control chart.

\section{Simulation results analysis}

In order to compare the control effect of traditional method tuning PID parameters and genetic algorithm tuning PID parameters, this paper used Matlab to write algorithm program, built actuator control block by Simulink, and then control simulation to the model respectively.

\subsection{Using step signal response to test}

As step signal response to displacement command, add step outside disturbance at the time of $0.2 \mathrm{~s}$ to observe the simulation results. Figure 7 is the traditional method tuning PID parameters and genetic algorithm tuning PID parameters control method results comparison chart.

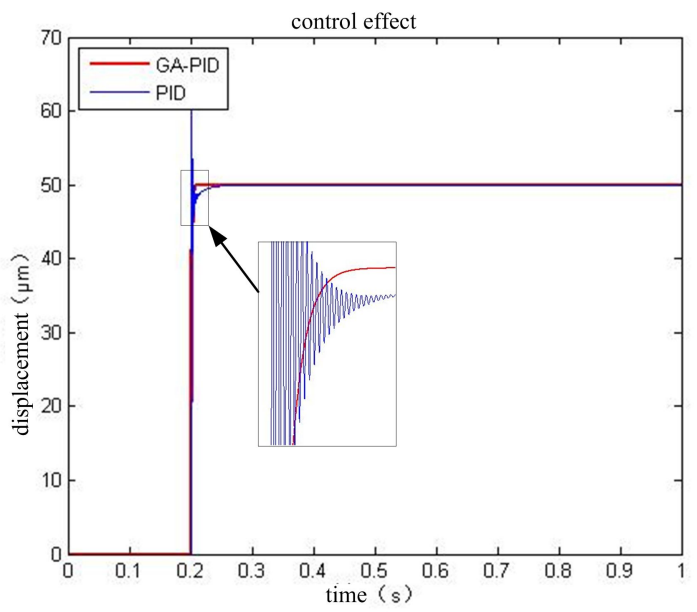

Figure 7. The control effects comparison between PID algorithm and GA-PID algorithm

By Figure 7 we can see, no matter what methods to control the system, the response of the system are fast, indicating that the system has fast response characteristics. When using traditional methods tuning PID parameters, the system overshoot is higher and the time to reach steady state is longer; When using genetic algorithm tuning PID parameters, the system can quickly reach a steady state, and there is no overshoot. This shows that the method of genetic algorithm tuning PID parameters can improve the deficiencies of traditional PID control, to achieve a better control effect.

\subsection{Using sinusoidal signal response to test}

Using sinusoidal signal as the control command signal, observe the effect of two control modes. Because the sine signal is constantly changing, so the controller must let the position tracking to signal, so as to achieve the purpose of real-time control. 


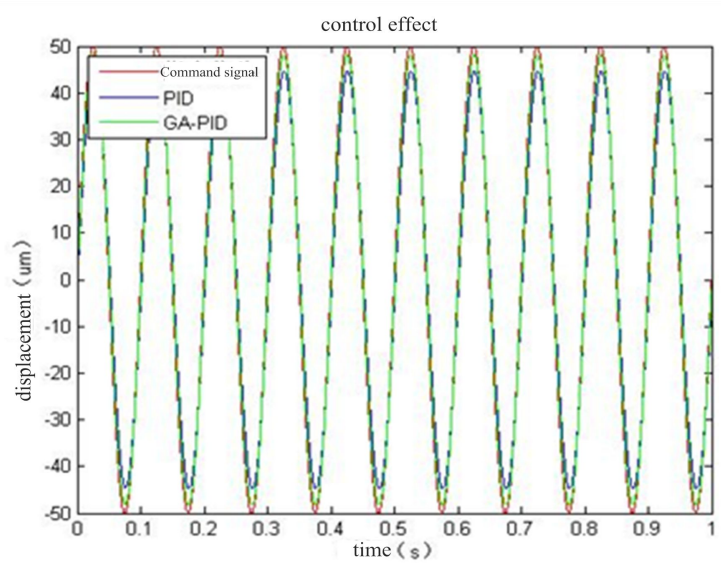

Figure 8. 10 Sinusoidal signal control effect comparison chart

Figure 8 can be seen the traditional method tuning PID parameters and genetic algorithm tuning PID parameters can achieve the purpose of location tracking, but the error of genetic algorithm tuning PID parameters is $4 \%$ and the traditional method tuning PID parameters is $10 \%$ by calculation. This shows that the genetic algorithm tuning PID parameters position tracking is stronger and control effect is better.

\section{Summary}

Through the establishment of the actuator control system mathematical model, the use of genetic algorithm tuning PID parameters to control the actuator, this paper got the renderings after control. Respectively using the step signal and the sinusoidal signal to simulate, and then comprise with traditional PID control, conclude: whatever signals using, genetic algorithm tuning PID control results are significantly better than the traditional method tuning PID parameters control. It shows that the method of genetic algorithm tuning PID parameters which designed at this paper is effective.

\section{References}

1. Zheng $\mathrm{H}, \mathrm{Wu} \mathrm{M}$, Wang C. Study on control of giant magnetostrictive piezoelectric hybrid actuator[C]. Guilin (2011)

2. Jalil M H A, Marzaki M H, Kasuan N, et al. Implementation of anti windup scheme on PID controller for regulating temperature of glycerin bleaching process. Shah Alam (2013)

3. Corcolle R, Daniel L, Bouillault F. Optimal Design of Magnetostrictive Composites: An Analytical Approach[J]. Magnetics, IEEE Transactions on. 44(1): 17-23 (2008)

4. Kiam H A, Chong G, Yun L. PID control system analysis, design, and technology[J]. Control Systems Technology, IEEE Transactions on, 13(4): 559 576 (2005)

5. Zhiqiang Z, Kun L, Yuqing H, et al. Model free analysis and tuning of PID controller[C]. Istanbul (2013)

6. Jian Z, Li L, Huanzhou L, et al. PID Tuning Based on Improved Quantum Genetic Algorithm[C]. Hangzhou (2013)

7. Shao X, Xiao L, Han C. Optimization of PID parameters based on genetic algorithm and interval algorithm[C]. Guilin (2009) 